\title{
Coexistence of cosmic-ray sidereal anisotropies originating in galactic space and at the heliomagnetospheric nose and tail boundaries, observed with muon detectors in the energy region of $60 \sim 100 \mathrm{GeV}$
}

\author{
K. Nagashima and Z. Fujii \\ Solar-Terrestrial Environment Laboratory, Nagoya University, Chikusa-ku, Nagoya 464-8601, Japan \\ (Received March 30, 2005; Revised June 5, 2006; Accepted June 24, 2006; Online published December 25, 2006)
}

\begin{abstract}
The coexistence of two kinds of cosmic-ray sidereal anisotropy was found by observations with underground muon telescopes in the energy region $(>\sim 200 \mathrm{GeV})$ in 1995: one is the galactic anisotropy with a deficit flux in the direction with right ascension $\alpha_{G}=12 \mathrm{hr}$ and declination $\delta_{G}=20^{\circ}$. The other is the excess flux from the heliomagnetospheric tail direction $\left(\alpha_{T} \simeq 6 \mathrm{hr}\right)$ and would be produced on the heliotail boundary where it is considered that the interaction between the galactic and solar magnetic fields could produce the cosmic-ray acceleration. On the other hand, another anisotropy of helioboundary origin from the helionose direction $(\alpha \simeq 18$ hr), being accompanied by the heliotail-in anisotropy, was found through the observations with neutron monitors in the low energy region $(\sim 20 \mathrm{GeV})$ in 2005 . These observations, however, lack information in the mid-energy region $(20 \sim 200 \mathrm{GeV})$. In order to bridge the absence of information, the cosmic-ray sidereal daily variations in the energy regions $(60 \sim 100 \mathrm{GeV})$ have been derived from the observations with muon telescopes and ion chambers on the ground in the period 1936-2003. It is shown that all the three anisotropies coexist in this energy region and are subject to their respective solar modulations. On the basis of these modulations, the characteristics of the anisotropies are determined through intercomparison with the observations in the high and low energy regions.
\end{abstract}

Key words: Cosmic-ray sidereal anisotropy, heliomagnetosphere (HMS), boundary of HMS, polarity of HMS, solar modulation.

\section{Introduction}

The galactic, helionose-in and heliotail-in anisotropies of cosmic rays (called $G-, H$ - and $T$-anisotropies or excess fluxes) produce sidereal daily variations called $G(t)$, $H(t)$ and $T(t)$ with their maxima at $\sim 0, \sim 18$ and $\sim 6 \mathrm{hr}$ respectively. $G(t)$ and $T(t)$ were found through observations with the underground muon telescopes at Hobart (median energy $E_{m}=184 \mathrm{GeV}$, geographic latitude $\lambda=43^{\circ} \mathrm{S}$, longitude $\phi=147^{\circ}$ E) and Sakashita $\left(E_{m}=300 \sim 400\right.$ $\left.\mathrm{GeV}, \lambda=36^{\circ} \mathrm{N}, \phi=138^{\circ} \mathrm{E}\right)$, while $H(t)$ was found together with $T(t)$ from observations with neutron monitors $\left(E_{m} \simeq 20 \mathrm{GeV}\right.$ ) on the ground. Detailed progress reports on the discovery of these anisotropies were presented in several papers (Nagashima et al., 1995a, b, 1998, 2004, 2005, hereafter the latter three are called Refs. 1, 2 and 3 respectively). According to the reports in Refs. 2 and 3 , the anisotropies are subject to solar modulation. $G(t)$ becomes smaller in the positive polarity state of the solar magnetic field at the north pole than in the negative state and also becomes smaller in the active period of the solar cycle than in the quiet period. On the contrary, $T(t)$ and $H(t)$ do not show such a polarity dependence and become larger in the active period than in the quiet period. The simulation of solar modulation of a galactic anisotropy

Copyright (c) The Society of Geomagnetism and Earth, Planetary and Space Sciences (SGEPSS); The Seismological Society of Japan; The Volcanological Society of Japan; The Geodetic Society of Japan; The Japanese Society for Planetary Sciences; TERRAPUB. confirmed the occurrence of the observed polarity dependence as above and consequently denied the galactic origin of $T$ - and $H$-anisotropies (cf. Refs. 2 and 3). These nongalactic anisotropies are considered to be produced by the cosmic-ray acceleration on the nose and tail boundaries of the heliomagnetosphere (hereafter called HMS). The acceleration is active and intense on the tail-end boundary surface, especially in the high latitude region of the cylindrical tail boundary surface, in comparison with that on the nosehead boundary surface. In the present paper, which is the fourth paper of the series of papers Refs. 1, 2 and 3, such a qualitative nature of the anisotropies as above is reconfirmed and, at the same time, more quantitative characteristics of the anisotropies such as their energy spectrum and so forth, are obtained from long-term observations of cosmicray sidereal variations with 17 directional muon telescopes and 5 ion chambers on the ground in cooperation with those previously obtained by the neutron monitors and the underground muon telescopes.

\section{Analysis}

Cosmic-ray data used for the present analysis are those of multi-directional muon telescopes at Nagoya $\left(\lambda=35^{\circ}\right.$ $\left.\mathrm{N}, \phi=137^{\circ} \mathrm{E}\right)$ and ion chambers at Godhavn $\left(\lambda=69^{\circ}\right.$ $\left.\mathrm{N}, \phi=307^{\circ} \mathrm{E}\right)$, Yakutsk $\left(\lambda=62^{\circ} \mathrm{N}, \phi=130^{\circ} \mathrm{E}\right)$, Cheltenham $\left(\lambda=39^{\circ} \mathrm{N}, \phi=283^{\circ} \mathrm{E}\right)$, Huancayo $\left(\lambda=12^{\circ}\right.$ $\left.\mathrm{S}, \phi=285^{\circ} \mathrm{E}\right)$ and Christchurch $\left(\lambda=44^{\circ} \mathrm{S}, \phi=173^{\circ}\right.$ E). The overall observation period at these stations ran 
Table 1. Characteristics of the cosmic-ray detectors.

\begin{tabular}{|c|c|c|c|c|c|c|}
\hline \multirow{2}{*}{\multicolumn{2}{|c|}{$\begin{array}{c}\text { Station and } \\
\text { detector }\end{array}$}} & \multicolumn{2}{|c|}{ Center direction of viewing } & \multirow{2}{*}{$\begin{array}{c}\text { Median } \\
\text { energy } \\
\mathrm{E}_{m}(\mathrm{GeV})\end{array}$} & \multirow{2}{*}{$\begin{array}{c}\text { Counting } \\
\text { rates } \\
10^{4} \mathrm{c} / \mathrm{hr}\end{array}$} & \multirow[t]{2}{*}{ Period and references. } \\
\hline & & Latitude & Longitude & & & \\
\hline \multicolumn{7}{|c|}{ Muon Telescope } \\
\hline \multirow[t]{17}{*}{ Nagoya } & $\mathrm{V}$ & $35 \mathrm{~N}$ & 137 & 60 & 276 & \multirow[t]{2}{*}{$1971 \sim 2003$} \\
\hline & $\mathrm{N}$ & $65 \mathrm{~N}$ & 137 & 66 & 125 & \\
\hline & $\mathrm{S}$ & $5 \mathrm{~N}$ & 137 & 64 & 123 & \multirow{15}{*}{$\begin{array}{l}\text { Sekido et al., 1975, } \\
\text { Nagashima et al., 1978, } \\
\text { 1981a, 1984, } 1987 . \\
\text { Fujii et al., 1990, 1993, } \\
\text { 1996, 2002. }\end{array}$} \\
\hline & $\mathrm{E}$ & $30 \mathrm{~N}$ & 172 & 67 & 120 & \\
\hline & $\mathrm{W}$ & $30 \mathrm{~N}$ & 102 & 63 & 126 & \\
\hline & $\mathrm{NE}$ & $54 \mathrm{~N}$ & 187 & 73 & 58 & \\
\hline & NW & $54 \mathrm{~N}$ & 87 & 68 & 62 & \\
\hline & SE & $5 \mathrm{~N}$ & 164 & 71 & 58 & \\
\hline & SW & $5 \mathrm{~N}$ & 110 & 67 & 60 & \\
\hline & $\mathrm{N}^{2}$ & $84 \mathrm{~N}$ & 137 & 87 & 61 & \\
\hline & $S^{2}$ & $14 \mathrm{~S}$ & 137 & 85 & 60 & \\
\hline & $E^{2}$ & $22 \mathrm{~N}$ & 192 & 91 & 58 & \\
\hline & $\mathrm{W}^{2}$ & $22 \mathrm{~N}$ & 82 & 84 & 62 & \\
\hline & $\mathrm{N}^{3}$ & $81 \mathrm{~N}$ & 317 & 110 & 14 & \\
\hline & $S^{3}$ & $29 \mathrm{~S}$ & 137 & 111 & 18 & \\
\hline & $E^{3}$ & $15 \mathrm{~N}$ & 205 & 119 & 17 & \\
\hline & $\mathrm{W}^{3}$ & $15 \mathrm{~N}$ & 69 & 110 & 18 & \\
\hline \multicolumn{7}{|c|}{ Ion Chamber } \\
\hline \multirow{2}{*}{\multicolumn{2}{|c|}{ Yakutsk (YAK) }} & $62 \mathrm{~N}$ & 130 & 67 & & $1953 \sim 1980$ \\
\hline & & & & & & Shafer and Shafer, 1984. \\
\hline \multicolumn{2}{|c|}{ Godhavn (GOD) } & $69 \mathrm{~N}$ & 307 & 67 & & \multirow{4}{*}{$\begin{array}{l}1936 \sim 1959 . \\
\text { Lange and Forbush, } 1948, \\
1957 . \\
\text { Beach and Forbush, } 1961 .\end{array}$} \\
\hline Cheltenh & (HE) & $39 \mathrm{~N}$ & 283 & 67 & & \\
\hline Huancay & JA) & $12 \mathrm{~S}$ & 285 & 67 & & \\
\hline Christch & CHR) & $44 \mathrm{~S}$ & 173 & 67 & & \\
\hline
\end{tabular}

from 1936 to 2003. The characteristics of these detectors are listed in Table 1 . The sidereal daily variation $i(t)$ of each detector is derived every calendar year from those data which do not contain incomplete solar daily variations nor abnormally large fluctuations such as GLE (Ground Level Enhancement of cosmic rays). $i(t)$ is influenced by the annual variation (1 cycle/year) of the solar daily variation (365 cycles/year) connected with the orbital motion of the Earth around the Sun. This influence is eliminated by the method using the anti-sidereal daily variations (364 cycles/year), (cf. Ref. 1). In the case of the ion chamber data, their longterm drift is also eliminated.

In order to study the origin of the cosmic-ray anisotropy responsible for the sidereal variation by means of its solar modulation, $i(t)$ is classified into four periods $(Y=$ $A P, Q P, A N$ and $Q N$ ) in which $A$ and $Q$ express active and quiet periods in the solar cycle divided as equally as possible in considering solar activity, whereas $P$ and $N$ are periods of the positive and negative polarity state of the solar magnetic field at the north pole quoted from Howard (1974) and Solar Geophysical Data, U.S. Dept. of Commerce. The combined periods in the above express 'Active and Positive' period, 'Quiet and Positive' period, and so forth. $i(t)$ is specified by $i(X, Y, Z)$ in which $Z$ is the observation year and $X$ is the abbreviation of the detector or station name in Table 1 or ' $M U O N$ ' and 'ION' of the muon telescope and the ion chamber. The average of $i(X, Y, Z) \mathrm{s}$ in $Y$ period is expressed as $I(X, Y)$. The variation averaged over all the periods is expressed as $I(X, A L L)$, and in the case of no specification of $X$ required, it is expressed as $I(t)$ or $I(t, Y)$. The expression for $I(X, Y)$ is applied to $G(t), T(t)$ and $H(t)$ in the specified period.

The sidereal daily variation is connected with the sidereal anisotropy just outside the geomagnetosphere (GMS) by the so-called coupling coefficient which depends on the energy spectrum of the anisotropy, and the cosmic-ray deflection in GMS and attenuation in the atmosphere (cf. Yasue et al., 1982; Fujimoto et al., 1984, the latter is called Ref. 4 hereafter). By using the coupling coefficients, the sidereal anisotropy is derived inversely from the observed sidereal daily variations.

\section{Sidereal Daily Variation}

Figure 1 shows latitude dependences of $I(X, A L L) \mathrm{s}$ of the muon telescopes $(X=M U O N)$ and the ion chambers $(X=I O N) . I(M U O N, A L L)$ s on the left-hand column show a clear peak at $\sim 6 \mathrm{hr}$, which expresses $T(t)$ produced by $T$ anisotropy. The peak accompanies a small hump on the left side whose physical meaning will be clarified later. With the increase of Northern latitude, the peak and hump are degenerated into a single broad peak at $\sim 6 \mathrm{hr}$. In the case of the variations of the ion chambers on the right side, $I(\mathrm{CHE}$, $A L L)$ at $\lambda=39^{\circ} \mathrm{N}$ has the typical peak and hump similar to those of $I(V, A L L)$ on the left side. In the equatorial region, however, I (HUA, ALL) does not show a sharp peak like $I\left(S^{2}, A L L\right)$ on the left. This would be due to the blunting of the peak caused by the wide spread of geomagnetic deflections of cosmic rays with different energies, which 


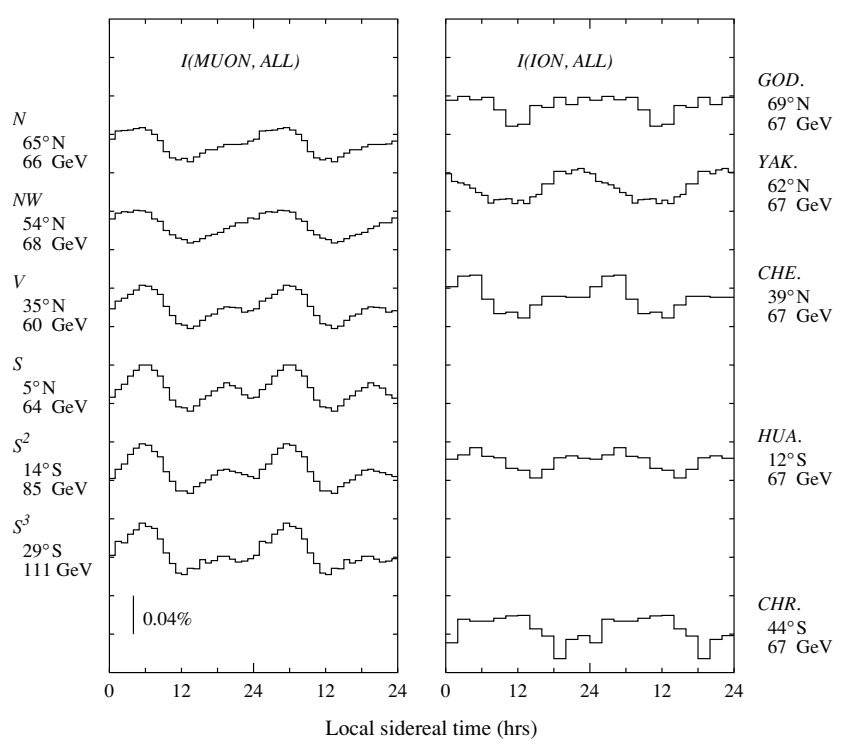

Fig. 1. Latitude dependence of cosmic-ray sidereal daily variations $I(X$, $A L L)$ s of muon telescopes on the left column and ion chambers on the right in ' $A L L$ ' period. The 24-hour variation is repeatedly plotted so as to extend for a 2-day period. The viewing directions $\left(\lambda_{c}\right)$ of detectors and the median energy $\left(E_{m}\right)$ of cosmic rays are attached to each variation, (cf. Table 1).

is especially remarkable at Huancayo (Inoue et al., 1983). At Southern high latitudes, I (CHR, ALL) shows the minimum at $\sim 18 \mathrm{hr}$ where the hump is observed in the Northern Hemisphere. This means that $I(C H R, A L L)$ contains only $T(t)$. Although this variation does not show a sharp peak characteristic of the typical $T(t)$, its sole existence is consistent with that of the variation observed with the underground muon-telescope at Hobart $\left(\lambda=43^{\circ} \mathrm{S}\right)$, (Jacklyn, 1986). The disappearance of the hump in the Southern high latitudes can be also inferred from the gradual decrease of the hump of the muon component with the increase of the Southern latitude (cf. Fig. 1). At Northern high latitudes, on the other hand, $I(G O D, A L L)$ and $I(Y A K, A L L)$ are different in shape from $I(N, A L L)$ and $I(N W, A L L)$ of the muon telescopes; the variation at Yakutsk has a peak at $\sim 20 \mathrm{hr}$ contrary to the peak ( $\sim 6 \mathrm{hr})$ of the muon telescopes, and the variation at Godhavn does not show any clear peak. The reason of such differences will be discussed later in relation to the existence of $G$-anisotropy.

\section{Solar Modulation}

Sidereal daily variations are subject to solar modulation. As an example of the modulation, $I(S, A L L)$ of the muon $S$-telescope in Fig. 1 is decomposed into $I(S, Y)$ s in four periods $(Y=A P, Q P, A N, Q N)$, as shown in Fig. 2. $I(S, A P)$ shows a diurnal variation with an extremely large peak at $\sim 6 \mathrm{hr}$ due to $T$-anisotropy, whereas $I(S, Y \neq A P)$ s are very small semidiurnal variations with the maxima at $\sim 6$ and $\sim 18$ hr. Such semidiurnal variations were not observed at Sakashita underground station (cf. Refs. 1 and 2), and their physical interpretation was left unsolved until $H$-anisotropy from the direction of $\sim 18 \mathrm{hr}$ was discovered by the recent analysis of neutron monitor data (cf. Ref. 3). Owing to the findings, the semidiurnal variation of $I(X, Y \neq A P)$ can be interpreted as being due to the superposition of yearly

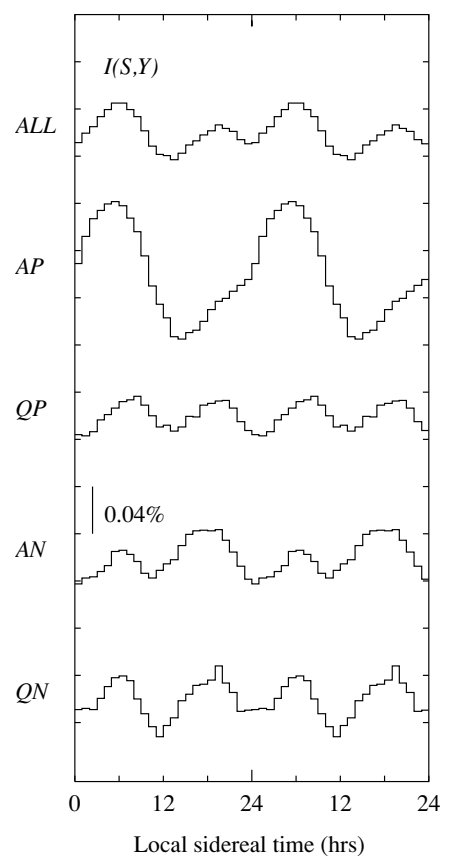

Fig. 2. Sidereal variations $I(S, Y) \mathrm{s}$ of $S$-telescope in $Y=A L L$ (1971-2003), AP (1971-'73, '78, '91-'93, '99 - 2000), QP (1974-'77, '94-'97), AN (1980 - '83, '88, '89, 2002-2003) and QN (1984-'87).

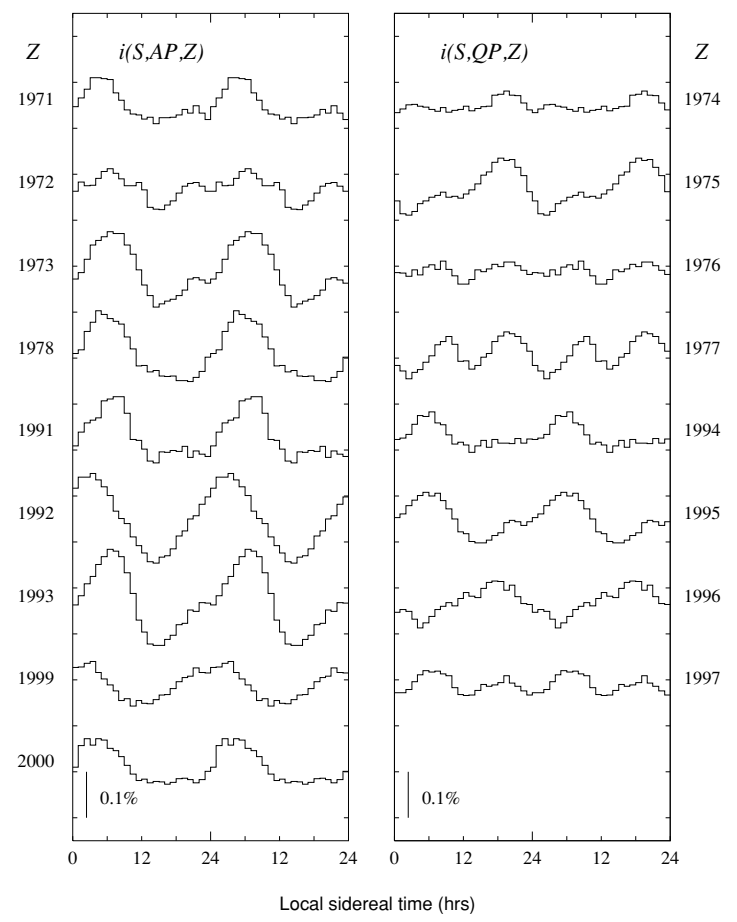

Fig. 3. Yearly component variations $i(S, Y, Z) \mathrm{s}$ of $I(S, Y)$ in $Y=A P$ and $Q P$.

variations $i(X, Y, Z) \mathrm{s}$ in $Y \neq A P$ period, which contain $H(t)$ and/or $T(t)$ produced by $H$ - and/or $T$-anisotropies, as follows. Fig. 3 shows all the yearly variations $i(S, A P$, $Z)$ s and $i(S, Q P, Z)$ s contained respectively in $I(S, A P)$ and $I(S, Q P)$ in Fig. 2. In $Q P$ period, all the variations show one or two peaks at $\sim 6$ and/or $\sim 18 \mathrm{hr}$ which can be regarded as being due to $T(t)$ and/or $H(t)$. In the case of two peaks, the variations are generally small. This would 


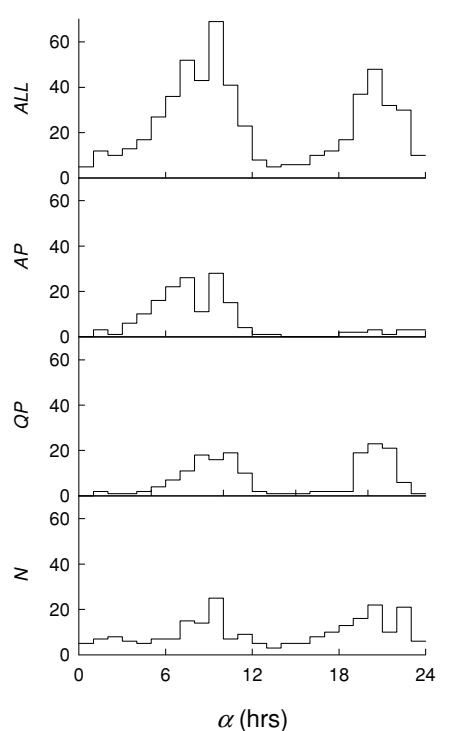

Fig. 4. Occurrence frequency $f_{Y}(\alpha)$ of the right ascension $\alpha$ of the direction of the anisotropy derived from the time of maximum of $T(t)$ and/or $H(t)$ among $i(X, Y, Z)$ s in $Y=A L L, A P, Q P$ and $N$, (see the text).

be mainly due to the interaction between $T(t)$ and $H(t)$ with the mutually opposite maximum phases. Therefore, the true magnitude of each variation cannot be observed. Even in the case of a single peak, the magnitude of the variation might be reduced by the other variation submerged beneath the bottom. Owing to the superposition of $i(S$, $Q P, Z)$ s, their average $I(S, Q P)$ in Fig. 2 shows a very small semidiurnal variation. On the other hand, almost all $i(S, A P, Z) \mathrm{s}$ in Fig. 3 show a large single peak of $T(t)$ at $\sim 6 \mathrm{hr}$, indicating that $A P$ period activates $T(t)$ and/or inactivates $H(t)$. As mentioned in the Introduction, the activation of $T(t)$ in $A P$ period was also observed with the neutron monitors and the underground muon telescopes in the low $(\sim 20 \mathrm{GeV})$ and high $(\sim 300 \mathrm{GeV})$ energy regions, respectively. This phenomenon was considered to be due to the arrival of cosmic rays being most severely accelerated at the polar region $\left(|\lambda| \gg 0^{\circ}\right)$ of the cylindrical surface of the heliotail boundary in active periods in the positive polarity state (cf. Ref. 3). Incidentally, that the largest $T(t)$ represented by $i(S, A P, 1992)$ and $i(S, A P, 1993)$ in Fig. 3 is about $0.2 \%$ in magnitude which amounts to half the solar diurnal variations of the $S$-telescope in the same periods. If there were no interaction between $T(t)$ and $H(t)$, the magnitude of $T(t)$ would become much larger than $0.2 \%$.

The right ascension $(\alpha)$ of the direction of $T$ - and $H$ anisotropies on the boundary of GMS can be approximately inferred from the maximum time of $T(t)$ and/or $H(t)$ in $i(X, Y, Z)$ with the aid of the coupling coefficient of an $X$ telescope for an energy-independent cosmic-ray spectrum (cf. Ref. 4). The occurrence frequency $f_{Y}(\alpha)$ of $\alpha$ in the $Y$ period is shown in Fig. 4, in which $A N$ and $Q N$ periods are integrated into the $N$ period as the latter period contains only four years. Although $\alpha$ is only roughly estimated owing to the unknown energy spectrum, it is clear that $f_{Y}(\alpha)$ in the $Y$ period is separated into two groups $f_{Y}^{T}(\alpha)$ and $f_{Y}^{H}(\alpha) . f_{A P}^{T}(\alpha)$ is larger in the extreme than $f_{A P}^{H}(\alpha)$,

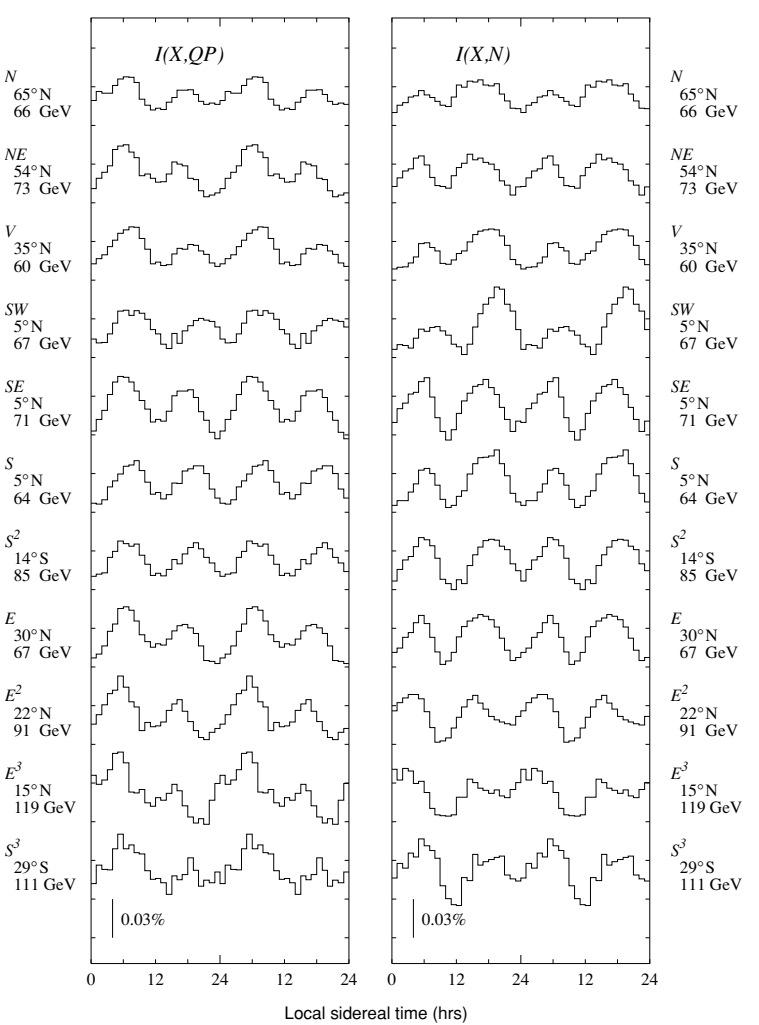

Fig. 5. $I(X, Y) \mathrm{s}$ in $Y=Q P$ and $N$ period, whose distribution is arranged so that adjoining variations are alike in shape as possible.

while $f_{Q P}^{T}(\alpha)$ is still somewhat greater than $f_{Q P}^{H}(\alpha)$. This means that the $P$-period activates $T$-flux (or anisotropy) and/or inactivates $H$-flux and, furthermore, the activation of $T$-flux is strongest in the $A$-period. On the contrary, the $N$-period tends to activate $H$-flux and/or inactivate $T$-flux as $f_{N}^{H}(\alpha)$ becomes larger than $f_{N}^{T}(\alpha)$. Such a polarity-state dependence shows a good agreement with that observed with the neutron monitors (cf. Ref. 3).

It is further noted that the criterion of the classification between $A$ and $Q$ periods is not definite. The present classification was made to obtain $I(X, Y) \mathrm{s}$ in Fig. 2 without any information on yearly variations $i(X, Y, Z) \mathrm{s}$ in Fig. 3 . Nevertheless, the clear separation between $i(S, A P, Z) \mathrm{s}$ and $i(S, Q P, Z)$ s happened to be obtained through the classification shown in the caption in Fig. 2. One can change the criterion according to the behavior of $i(X, Y, Z)$. It should also be mentioned that in the present analysis the transition from the positive to negative state or vice versa was determined by the polarity reversal of the solar magnetic field at the north pole. Strictly speaking, the time of the transition should be delayed for the present analysis by about one year or so, which is required for the pervasion of the information of the reversal over whole region of HMS. But, the alteration of the time would not affect the polarity dependence of the sidereal variation in the present analysis so much.

\section{Nose-in and Tail-in Anisotropies}

The nature of $H$ - and $T$-anisotropies is inferred from the geographic latitude and longitude distributions of $I(X, Y) \mathrm{s}$ or $i(X, Y, Z)$ s of 17 muon telescopes in $Y=A P, Q P$ and the $N$ period. In Fig. 5, $I(X, Y) \mathrm{s}$ in $Y=Q P$ and $N$ 

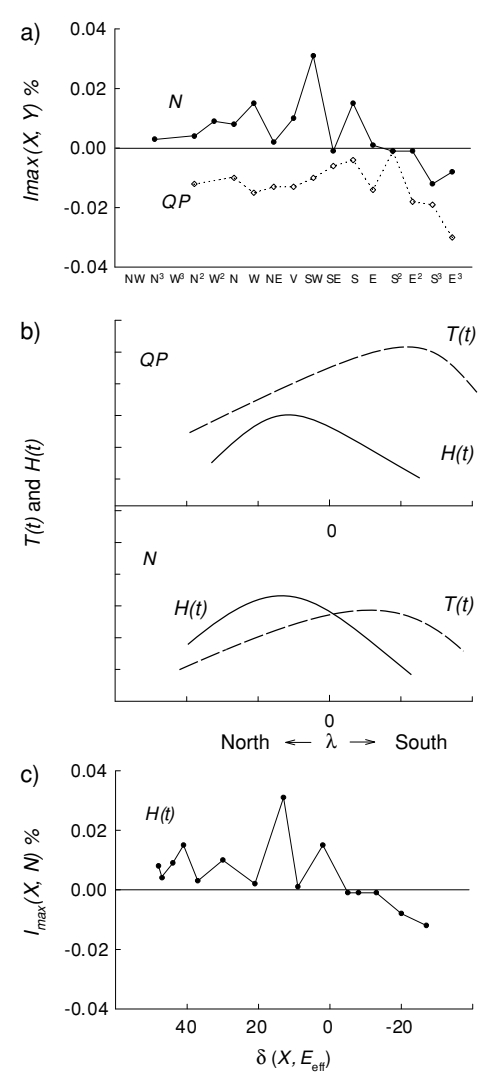

Fig. 6. a). $X$-dependence of the difference $\Delta I_{\max }(X, Y)$ between the maximum values of $H(t)$ and $T(t)$ in $I(X, Y)$ in $Y=Q P$ and $N$. b). Schematic representation of the latitude distributions of $H(t)$ and $T(t)$ in the $Q P$ and $N$ periods, which would produce $\Delta I_{\max }(X, Y) \mathrm{s}$ in Fig. 6(a). c). Distribution of $\Delta I_{\max }(X, N)$ as a function of $\delta\left(X, E_{\text {eff }}=\right.$ $65 \mathrm{GeV}$ ) which is the declination of the geomagnetic asymptotic orbital direction of cosmic rays with the effective energy $\left(E_{\text {eff }}\right)$ observed with $X$-telescope.

periods are distributed so that neighboring variations are alike in shape in order to facilitate the following discussion. As can be seen in the figure, the semidiurnal variations of $I(X, Y)$ s seem similar to each other at a glance, but they show the following polarity-state $(P$ or $N)$ dependence. The difference $\Delta I_{\max }(X, Y)$ between the maximum values of $H(X, Y)$ and $T(X, Y)$ is negative in the $Y=Q P$ period for all the $X$-telescopes, whereas it is positive in the $N$ period except for that of $S^{3}, S^{2}, E^{3}$ and $E^{2}$ telescopes pointing toward the equatorial region or the Southern Hemisphere, as shown in Fig. 6(a). Christchurch $\left(\lambda=44^{\circ} \mathrm{S}\right)$ and Hobart $\left(\lambda=43^{\circ} S\right)$ in the Southern Hemisphere also always observe $\Delta I_{\max }(X, Y)$ of negative value as $H(X, Y)$ is zero in the region (cf. Fig. 1 and Jacklyn, 1986). Such a polarity dependence of $\Delta I_{\max }(X, Y)$ s can be explained if $H$ - and $T$-anisotropies have, respectively, directions with positive and negative declinations, i.e. $\delta_{H}>0^{\circ}$ and $\delta_{T}<0^{\circ}$, and produce the polarity-dependent $H(t)$ and $T(t)$ as shown by the schematic diagrams in Fig. 6(b). The declination $\left(\delta_{H}\right)$ of $H$-anisotropy can be inferred from $\Delta I_{\max }(X, N)$ s on the assumption that the anisotropy has a monochromatic energy called the effective energy $\left(E_{\text {eff }}\right)$. In this case, $\Delta I_{\max }(X, N)$ is expressed on the boundary of GMS as a function of the declination $\delta\left(X, E_{\text {eff }}\right)$ of the geomagnetic asymptotic orbital direction of cosmic rays with $E_{\text {eff }}$ observed by the

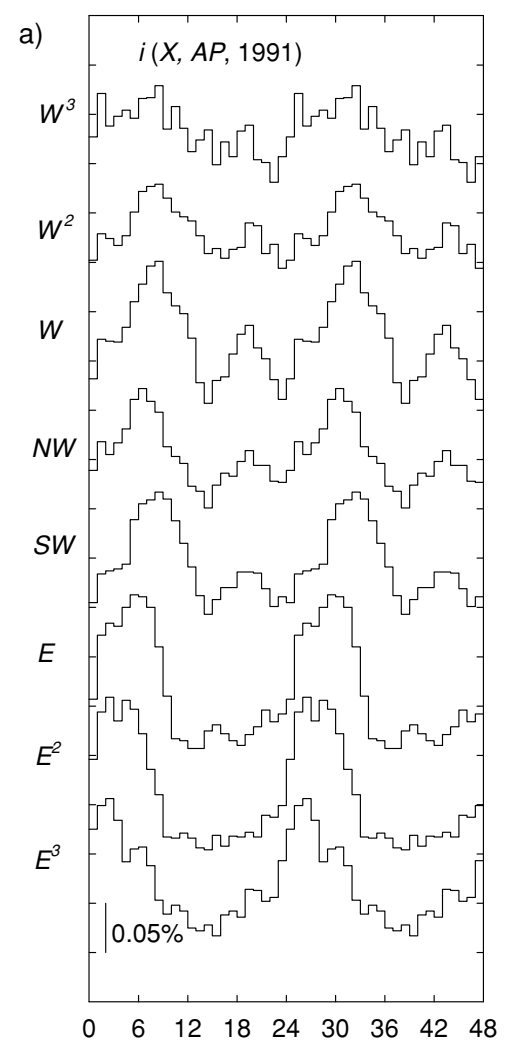

b) $10^{-2} \% \quad$ Local sidereal time (hrs)

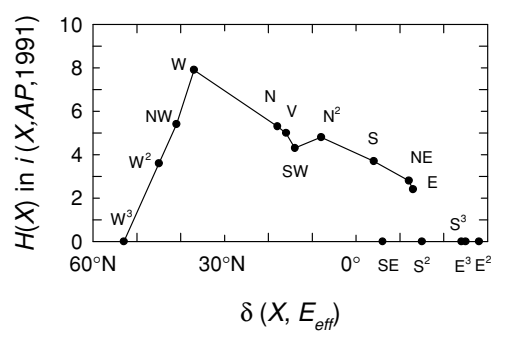

Fig. 7. a). $i(X, A P, 1991)$ s showing the rise and fall of $H(X, A P, 1991)$ at $\sim 18 \mathrm{hr}$ depending on $X$. b). Distribution of the magnitude of $H(X, A P$, $1991)$ as a function of $\delta\left(X, E_{\text {eff }}=35 \mathrm{GeV}\right)$, (cf. Fig. 6).

$X$-telescopes. $\delta_{H}$ is determined by choosing $E_{\text {eff }}$ so that $\Delta I_{\text {max }}(X, N)$ s show a systematic distribution of $\delta\left(X, E_{\mathrm{eff}}\right)$. Fig. 6(c) shows that the best distribution is obtained by $E_{\text {eff }}=65 \mathrm{GeV}$. As the maximum of the distribution is at $\sim 13^{\circ}, \delta_{H}$ and $E_{\text {eff }}$ of $H$-anisotropy are $\sim 13^{\circ}$ and $\sim 65 \mathrm{GeV}$, respectively. Practically, however, the energy spectrum is not monochromatic but of the power-exponent type with the exponent $\gamma$. Thus $\Delta I_{\max }(X, N)$ is expressed as a function of $\delta(X, \gamma)$ derived from the coupling coefficient for the $X$ telescopes. Although the $\delta$-distribution of $\Delta I_{\max }(X, N) \mathrm{s}$ is changed by this alteration, $\delta_{H}$ does not change so much for any reasonable energy spectrum, because $\delta(S W, E)$ for the $S W$-telescope, which observes the largest $\Delta I_{\max }$ in any case, is confined in a narrow range of $14^{\circ} \sim 17^{\circ}$ in the wide energy region $(25 \sim 150 \mathrm{GeV})$. In Fig. 7(a) another example is given for the estimation of $\delta_{H}$ and $E_{\text {eff }}$ of the $H$-anisotropy. In these variations, $H(X, A P, 1991)$ first appears at $X=E$ in the equatorial region as a tiny protuberance at $\sim 18 \mathrm{hr}$, then gradually increases toward the Northern regions and finally reaches its maximum at $X=W$. Then $\delta_{H}$ can be estimated 


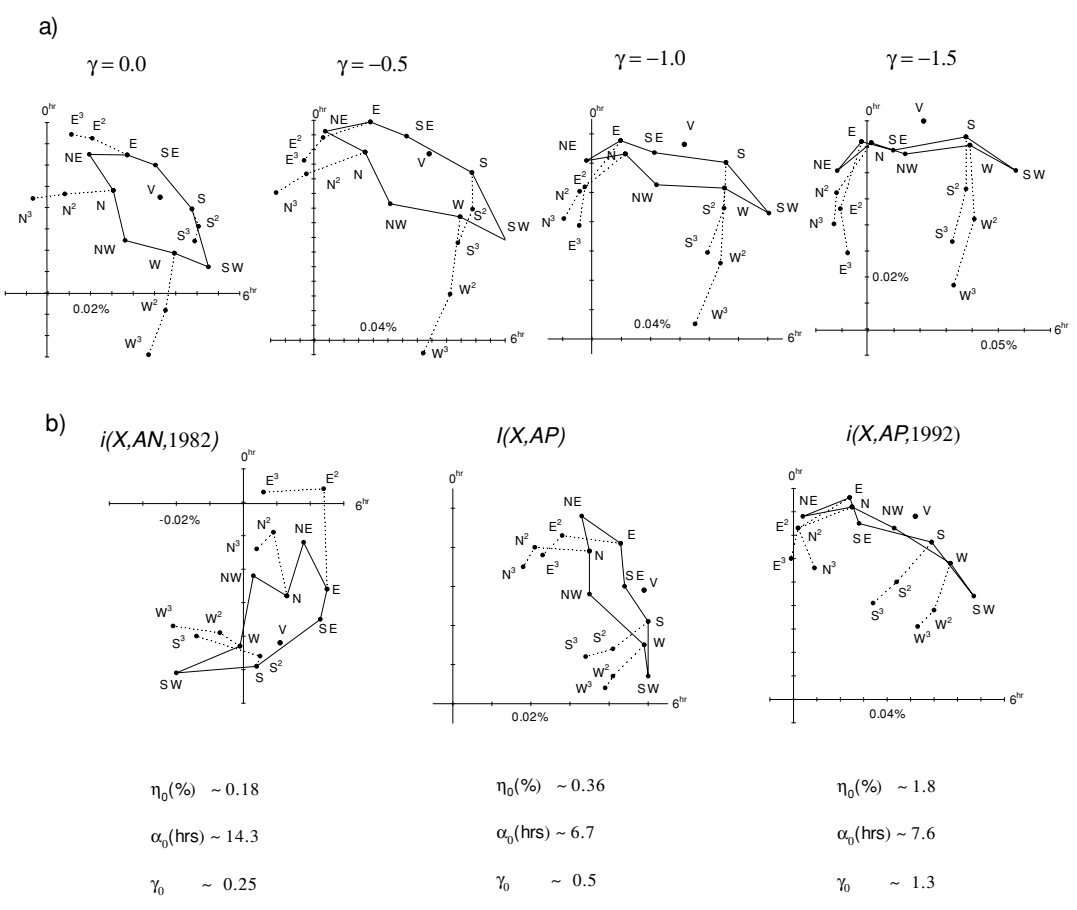

Fig. 8. a). Configuration $C_{\gamma_{0}}$ of the simulated first harmonic vectors (amplitude $r$ and phase $\phi_{\max }$ ) of the sidereal variations of 17 muon telescopes produced by the anisotropy from $\phi=90^{\circ}$ with the spectrum defined by Eq. (1) with $\eta_{0}=1.0$ for several $\gamma_{0}$ s. $\phi$ is measured clockwise from the top. Each vector is indicated by a point at $r(X)$ and $\phi_{\max }(X)$ with the label $X$ and connected with neighboring points by solid or dashed line. b). Three examples of observed configuration $C(Y, Z)$ of $i(X, Y, Z)$ and $C(Y)$ of $I(X, Y)$. The magnitude $\eta_{0}$ (double the amplitude $\left.r_{0}\right)$, the power exponent $\left(\gamma_{0}\right)$ and the right ascension $\left(\alpha_{0}\right)$ of the direction of the estimated anisotropy is listed under the corresponding $C(Y, Z)$.

by choosing $E_{\text {eff }}$ so that the $H(X, A P, 1991)$ s show a smooth distribution of $\delta\left(X, E_{\mathrm{eff}}\right)$. The best distribution is obtained for $E_{\text {eff }} \simeq 35 \mathrm{GeV}$. In Fig. 7(b) the peak appears at $\delta(W$, $35 \mathrm{GeV})=37^{\circ}$. Therefore $\delta_{H}$ is considered to be $\sim 37^{\circ}$, but would vary in the range of $24^{\circ} \sim 41^{\circ}$ for the same reason as is mentioned in the treatment of the non-monochromatic energy spectrum. In the present case, there is another uncertainty for the estimation of $\delta_{H}$. As can be seen in Fig. 7(b), the peak of the distribution might be located at the intermediate of $\delta(W, 35 \mathrm{GeV})$ and $\delta(N, 35 \mathrm{GeV})$. Even if we take the middle of these two points as the peak point, $\delta_{H}$ is $28^{\circ}$ and included in the range mentioned above.

The above analyses were made in terms of the coexistence of $H(t)$ and $T(t)$. If either one of these variations dominates extremely over the other, the usual harmonic analysis of the variations can be applied for the determination of the anisotropy with the energy spectrum given by

$$
S_{0}(E)=\eta_{0} \cdot(E / 10 \mathrm{GeV})^{\gamma_{0}} .
$$

The harmonic dials in Fig. 8(a) show the first harmonic vectors with amplitude $r$ and phase $\phi_{\max }\left(=15^{\circ} \times t_{\max }\right)$ of the simulated variations produced by the anisotropy with $\eta_{0}=1$ from the direction $\left(\phi_{0}\right)$ of $6 \mathrm{hr}$ for the specified $\gamma_{0}$ (cf. Ref. 4). The phase is measured clockwise from a 0-hour axis (upward direction) to a 6-hour axis (rightward direction). In order to emphasize the relative configuration $C_{\gamma_{0}}$ of 17 vectors, each vector is indicated by a point at $r(X)$ and $\phi_{\max }(X)$ with the label $X$ and connected with neighboring points by a solid or dashed line like a constellation in the sky. Corresponding to $C_{\gamma_{0}}$, the observed configuration is expressed as $C(X, Y)$ or $C(Y)$ and shown in Fig. 8(b). $C(A P)$ at the center is for $I(X, A P)$ s whose shapes can be inferred from $I(S, A P)$ in Fig. 2. Although this variation contains the unsuitable variations such as $i(X, A P, 1972)$ for $T(t)$ as can be seen in Fig. 3, it does not affect $C(A P)$ so much. $C(A P$, $1992)$ on the right side is for $i(X, A P, 1992) \mathrm{s}$ whose shapes can be inferred from an example $i(S, A P, 1992)$ in Fig. 3. Although these variations belong to the $A P$ group, $C(A P$, $1992)$ is clearly different from $C(A P)$ at the center. $C(A N$, $1982)$ on the left is for $i(X, A N, 1982)$ s which express approximately $H(t)$. This configuration indicates the hardest spectrum among the three. The magnitude $\eta_{0}$ (double the amplitude $r_{0}$ of the first harmonic component), the power exponent $\left(\gamma_{0}\right)$ and the right ascension $\left(\alpha_{0}\right)$ of the direction of the anisotropy are obtained by comparing $C(Y, Z)$ with $C_{\gamma_{0}}$, (cf. Ref. 4). The estimated anisotropy is listed under the respective $C(Y, Z)$ s. These results suggest that the spectra of the anisotropies responsible for $T(t)$ and $H(t)$ are variable in magnitude, hardness, and direction. The variable direction is inferable also from the broad distribution of $\alpha(Y)$ in Fig. 4 or the dispersion of the peak of $T(t)$ and $H(t)$ in Fig. 3. As $H$ - and $T$-anisotropies are considered to be produced by cosmic-ray acceleration on the boundary surface of HMS, their magnitude, energy spectrum and direction would be variable in time depending on the variable location and strength of cosmic-ray acceleration on the boundary. These anisotropies produce the averaged $T(t)$ and $H(t)$ with their maxima at $\sim 6$ and $\sim 18 \mathrm{hr}$, respectively. The power exponent $\left(\gamma_{0}\right)$ of the averaged energy spectrum of $T$-anisotropy would be $\sim-0.5$ derived from $I(X, A P)$ in Fig. 8. The average spectrum of $H$-anisotropy is not certain as the isolated $H(t)$ s are very small in number. This spectrum will be discussed later by synthesizing all the in- 


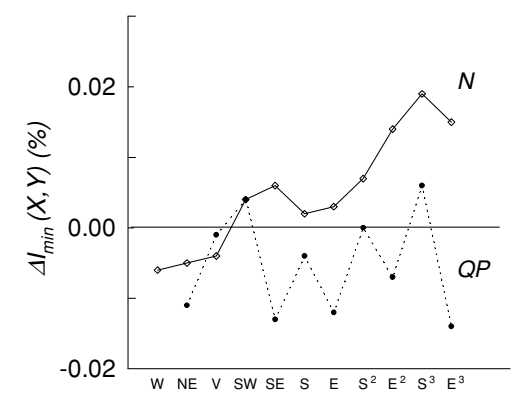

Fig. 9. $X$-dependence of the difference $\Delta I_{\min }(X, Y \mid \sim 0$ hr) between $I_{\min }(X, Y \mid \sim 0 \mathrm{hr})$ and $I_{\min }(X, Y \mid \sim 12 \mathrm{hr})$ in $Y=Q P$ and $N$, (see the text).

formation including those of the neutron monitors and the underground muon telescopes in low and high energy regions. It is noted that the present harmonic analysis cannot determine the declination $(\delta)$ of the anisotropy because the harmonic component of the second order for the representation of north-south asymmetric latitude distribution of the variation is not taken into consideration owing to insufficient information of the variations in the Southern Hemisphere.

\section{Galactic Anisotropy}

As pointed out previously, the semidiurnal variations in $Q P$ and $N$ periods in Fig. 5 express the coexisting states of $T(t)$ and $H(t)$. The states in the two periods are not identical: $T(t)$ is dominant over the $H(t)$ in $Q P$ period and vice versa in the $N$ period (cf. Figs. 5 and 6). In addition to this difference, there is another difference between them. As can be seen in Fig. 5, the minimum value $I_{\min }(X$, $Q P \mid \sim 0 \mathrm{hr})$ of $I(X, Q P)$ in the midnight zone is equal to or deeper than $I_{\min }(X, Q P \mid \sim 12 \mathrm{hr})$ in the midday zone. In $N$-period, on the contrary, $I_{\min }(X, N \mid \sim 0 \mathrm{hr})$ is shallower than $I_{\min }(X, N \mid \sim 12 \mathrm{hr})$ for $X=E^{3}, S^{3}, E^{2}$ and so forth, whose viewing directions are in the equatorial region. The difference $\Delta I_{\min }(X, Y)$ between $I_{\min }(X, Y \mid \sim 0$ hr) and $I_{\min }(X, Y \mid \sim 12 \mathrm{hr})$ in the two periods is shown in Fig. 9. The positive value of $\Delta I_{\min }$ in $N$ period might be produced by the shifts of the two peaks of $T(t)$ and $H(t)$ toward the midnight zone due to the directional change of anisotropies. However, if this shift were to be produced, all the telescopes must observe a positive $\Delta I_{\min }$. This is against the observation that the positive value can be observed only by the telescopes with the comparatively high median energies $\left(E_{m}\right)$ and with the viewing direction toward low latitudes. Such a phenomenon could be produced by an additional variation $U(t)$ superposed on $T(t)$ and $H(t)$. $U(t)$ would have a hard energy spectrum and a plateau with its center and sink at $\sim 0$ and $\sim 12 \mathrm{hr}$ respectively and might be identifiable with $G(t)$. If such is the case, no appearance of the positive $\Delta I_{\min }$ in the $Q P$ period would be considered to be reasonable as $G(t)$ would not be observed in the positive polarity state owing to the severe solar modulation (cf. Ref. 3). In order to verify this assumption, an attempt is made to extract $G(t)$ from $I(X, N)$ by taking the difference $I(X, N-Q P)$ between $I(X, N)$ and $I(X, Q P)$ in Fig. 5, both of which are supposed to contain the semidiurnal vari-
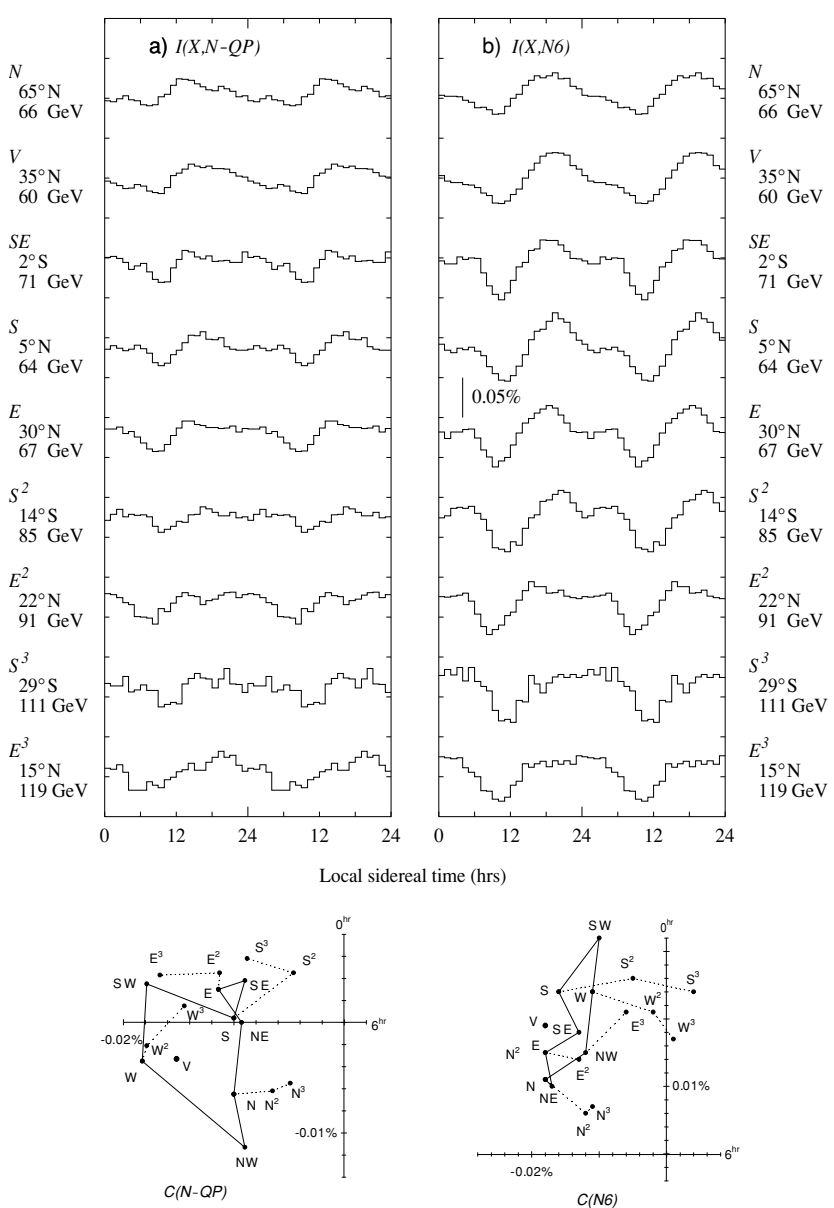

Fig. 10. a). The difference $I(X, N-Q P)$ between $I(X, N)$ and $I(X, Q P)$ in Fig. 5 is shown on the left-hand side, with $C(X, N-Q P)$ of the first harmonic vectors of $I(X, N-Q P)$ s at the bottom left-hand corner. b). The average $I(X, N 6)$ of $i(X, N, Z) \mathrm{s}$ in 6 years $(1981$, '83, '84, '87, ' 88, ' 89$)$ on the right-hand side and $C(N 6)$ of $I(X, N 6)$ s at the bottom right-hand corner.

ation similar to each other. Some $I(X, N-Q P)$ s are shown in Fig. 10(a). aAs anticipated, two explicit peaks characterizing $T(t)$ and $H(t)$ almost disappear in $I(X, N-Q P)$ s. Among these variations, those for $X=E^{3}, S^{3}, E^{2}, S^{2}$ and so forth in low latitudes are characterized with nearly horizontal plateau with its center at $\sim 0 \mathrm{hr}$ and the sink at $\sim 12 \mathrm{hr}$. Other variations, however, show the plateau with a declining slope toward late hours owing to the incomplete elimination of $H(t)$ at $\sim 18 \mathrm{hr}$. In order to examine the characteristics of $I(X, N-Q P) \mathrm{s}$, their configuration $C(N-Q P)$ is shown in Fig. 10(a). $C(N-Q P)$ is clearly different from the $C_{\gamma_{0}}$ 's of a single anisotropy in Fig. 8 and seems rather chaotic at a glance. However, the following characteristic relation between vectors can be seen: (1) $E$-, $E^{2}$ - and $E^{3}$ - vectors show phase lags behind the $V$-vector in opposition to the order of the longitudinal viewing directions of the eastward $\left(E, E^{2}, E^{3}\right)$ and the vertical telescopes, while (2) $S^{3}$ - and $S^{2}$-vectors also have phase lags behind the $W^{3}$ - and $W^{2}$-vectors. Such phase lags cannot be seen in the $C_{\gamma_{0}}$ 's in Fig. 8 and also cannot be realized by any combination of two kinds of configuration produced by the anisotropies with different amplitudes from different directions, unless their energy spectra are quite differ- 


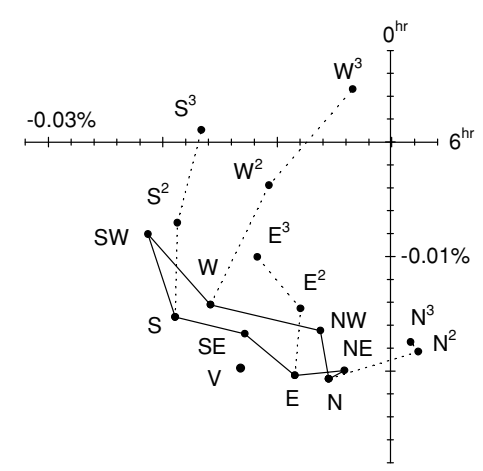

Fig. 11. The configuration of sidereal variation produced by two kinds of anisotropy with the hard energy spectrum given by Eq. (2) with $\eta_{U}=0.02, \gamma_{U}=0.5, E_{U}=200 \mathrm{GeV}$ and $\phi_{U}=0 \mathrm{hr}$ and with the soft spectrum given by Eq. (1) with $\eta_{0}=1.0, \gamma_{0}=-1.0$ and $\phi_{0}=18 \mathrm{hr}$.

ent in form from each other. Therefore, $\mathrm{C}(N-Q P)$ suggests the existence of $U(t)$ with an extremely different spectrum from the $H(t)$ 's and denies the previous supposition that $\Delta I_{\min }(X, N)$ s with positive values in Fig. 9 might be due to the shifts of the two peaks of $T(t)$ and $H(t)$ toward midnight zone. Encouraged by this result, a search was made to find the yearly variation of plateau type. Such events were found in 6 years of data (1981, '83, '84, '87, '88 and '89) in the $N$-period only. Some of their averages called $I(X, N 6)$ s are shown in Fig. 10(b). The variation of the typical plateau type is observed with $E^{3}, E^{2}$ and $S^{3}$ telescopes at low latitudes and is gradually hidden under the developing $H(t)$ and/or $T(t)$ with the increase of the Northern latitudes. $C(N 6)$ of the harmonic vectors of $I(X, N 6) \mathrm{s}$ is shown under Fig. 10(b). It has characteristics very similar to those of $\mathrm{C}(N-Q P)$ on the left-side. Attempts were made by trial and error to simulate such a configuration on the assumption that two kinds of anisotropy from 18 and $0 \mathrm{hr}$ directions have the respective spectra of $S_{0}(E)$ with $\gamma_{0}<0$ in Eq. (1) and $S_{U}(E)$ given by

$$
S_{U}(E)= \begin{cases}\eta_{U}(E / 10 \mathrm{GeV})^{\gamma_{U}>0} & \text { for } E \geq E_{U}, \\ 0 & \text { for } E<E_{U} .\end{cases}
$$

In Fig. 11 there is an example of the simulation, bearing the observed characteristic phase interrelationships obtained under the conditions shown in the figure caption. On the basis of the simulation, it is concluded that $C(N-Q P)$ and $C(N 6)$ in Fig. 10 are produced by $U(t)$ with a hard energy spectrum superposed on $H(t)$ and/or $T(t)$ with the soft spectrum. As $U(t)$ is the plateau-type variation with the minimum at $\sim 12 \mathrm{hr}$ produced by an anisotropy with a hard spectrum, it could be regarded as $G(t)$ which is observed only in the negative polarity state and has a magnitude of $0.05 \sim 0.06 \%$ for $X=E^{3}$ and $S^{3}$. The absence of $G(t)$ in the positive state in the low energy region is due to severe solar modulation (cf. Refs. 2 and 3).

It is emphasized that $G(t)$ can also be observed at Godhavn $\left(\lambda=69^{\circ} \mathrm{N}, \phi=307^{\circ}, 1939-1959\right)$ and Yakutsk $\left(\lambda=62^{\circ} \mathrm{N}, \phi=130^{\circ}, 1953-1980\right)$ in and close to the Arctic Circle, where $T(t)$ and/or $H(t)$ are considered to be very small, especially in the $N$ period. $I(G O D, A L L)$ and $I(Y A K, A L L)$ in Fig. 1 are decomposed into $I(X, P)$ and

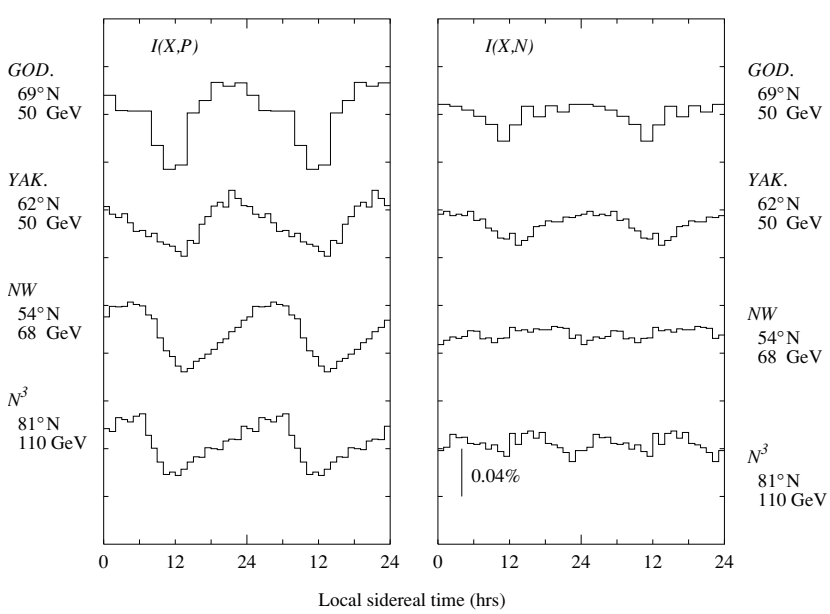

Fig. 12. Sidereal variations $I(G O D, Y), I(Y A K, Y), I(N W, Y)$ and $I\left(N^{3}, Y\right)$ in Northern high latitudes in the $Y=P$ and $N$ periods.

$I(X, N)$, respectively, as shown in Fig. 12. In spite of their almost independent observation periods, the corresponding variations at the two stations in Fig. 12 are almost the same, indicating their universality. As $I(G O D, N)$ and $I(Y A K, N)$ in $N$ period are plateau-type variations with the their center and sink at $\sim 0$ and $\sim 12 \mathrm{hr}$, respectively, they are considered to be produced by $G$-anisotropy. Furthermore, as the ratio of their magnitudes $(\sim 0.03 \%)$ at $\lambda_{\text {ION }} \simeq 65^{\circ} \mathrm{N}$ in Fig. 12 to that $(\sim 0.06 \%)$ of $G\left(E^{3}, N\right)$ at $\lambda_{E^{3}}=15^{\circ} \mathrm{N}$ in Fig. 10 is $\sim 0.5$ and nearly equal to the ratio $(\sim 0.44)$ of $\cos \lambda_{\mathrm{ION}} / \cos \lambda_{E^{3}}$, the variations in high and low latitude regions would be considered to be produced by a common source. However, $I\left(N^{3}, N\right)$ and $I(N W, N)$ in Fig. 12 observed with the muon telescopes in close vicinity of the ion chambers in latitude are different from $G(t)$. Such disagreement between the variations of the ion and muon groups is due to the difference of their trajectories of the asymptotic latitude $\lambda_{\text {asy }}(X, E)$ s. As can be seen in Fig. 13, the trajectories for the two groups lie almost in the same latitude range in the high energy region but they are separated from each other in the low energy region. On the other hand, as pointed out previously, the $G$-anisotropy has a hard energy spectrum and is observable only in the high energy region in the Northern Hemisphere, whereas $H$ - and $T$-anisotropies have a soft spectrum and are observable only in the low energy region. As $H(t)$ and $T(t)$ would be observed mostly in low latitudes in the $N$-period, the ion chambers can observe only $G(t)$. On the other hand, the muon telescopes observe not only $G(t)$ but also $H(t)$ and/or $T(t)$. Owing to their mutual interactions, the $N W$-telescope does not observe any significant variations, while the $N^{3}$-telescope observes a small but somewhat significant semidiurnal variation, as the energy region covered by its trajectory in low latitudes is wider than that of the $N W$-telescope (cf. Fig. 13).

With the polarity reversal from an $N$ to $P$ state, $G(t)$ disappears and $I(I O N, P)$ in Fig. 12 shows the maximum phase of $\sim 18 \mathrm{hr}$ different from that $(\sim 6 \mathrm{hr})$ of I (MUON, $P)$. This phase difference also could be explained with the aid of the above trajectory difference as follows. According to the previous analysis in Section 5, the declinations $\delta_{H}$ and $\delta_{T}$ of $H$ - and $T$-anisotropies are considered to be in 


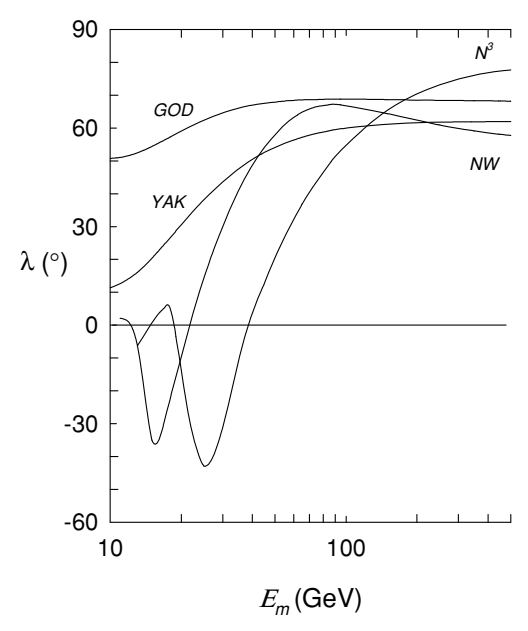

Fig. 13. Trajectories of asymptotic latitudes of cosmic rays for the four detectors in Fig. 12.

the Northern and Southern Hemispheres, respectively (cf. Fig. 6). As the trajectories at Godhavn and Yakutsk are far away from the direction $\left(\delta_{T}\right)$ of $T$-anisotropy, these stations could observe only $H(t)$ with its maximum at $\sim 18$ hr. On the contrary, those detectors such as $N W$ and $N^{3}$, whose trajectories lie in lower latitudes, could observe the superposed variation of $H(t)$ and $T(t)$ with its maximum at $\sim 6 \mathrm{hr}$ owing to the superiority of $T(t)$ over $H(t)$ in this region (cf. Fig. 13). As above, the observations of the ion chambers at high latitudes provide important information not only about the galactic anisotropy but also about solar modulation of the $T$ - and $H$-anisotropies.

\section{Discussion}

Fig. 14 shows $I(N M, Y) \mathrm{s}, I(N A G, Y) \mathrm{s}$ and $I(S A K, Y) \mathrm{s}$ in the low $(L O)$, middle $(M I)$ and high $(H I)$ energy regions observed respectively by the neutron monitor $(N M)$ and the muon telescopes at Nagoya $(N A G)$ and Sakashita $(S A K)$. These variations are presented in order to compare their solar modulations in wide energy regions. For this purpose, it would be preferable to use the component variations $T(X, Y) \mathrm{s}, H(X, Y) \mathrm{s}$ and $G(X, Y)$ s contained in $I(X, Y) \mathrm{s}$, but it is difficult to do so, because, although these components were obtained for $X=N M$ and $S A K$ in Refs. 3 and 2, they can hardly be separated from each other for $X=N A G$ except in the $Y=A P$ period owing to their strong interaction.

In $A L L$-period in Fig. 14, $I(X, A L L)$ s in the three energy regions change their shape depending on the relative contribution of $T(t), H(t)$ and $G(t)$. A clear difference can be seen in the location of the hump, which is on the left side of the peak at $\sim 6 \mathrm{hr}$ in $H I$ - and $M I$-regions but on the right side in $L O$-region. Consequently, the minimum of $I(X$, $A L L)$ appears at $\sim 12 \mathrm{hr}$ in $H I$ - and $M I$-regions, whereas it is at $\sim 0 \mathrm{hr}$ in $L O$-region, where the center of $G(t)$ is expected to be found. In other words, the minimum of $I(X$, $A L L)$ at $\sim 12 \mathrm{hr}$ (or $\sim 0 \mathrm{hr}$ ) indicates the existence (or nonexistence) of $G(X, A L L)$. This criterion is applicable also to $I(X, Y) \mathrm{s}$ in $Y(=A P, Q P, A N, Q N)$ period except for $I(N M$, $A P)$ and $I(N A G, A P)$. As can be seen in Fig. 14, $I(S A K$,

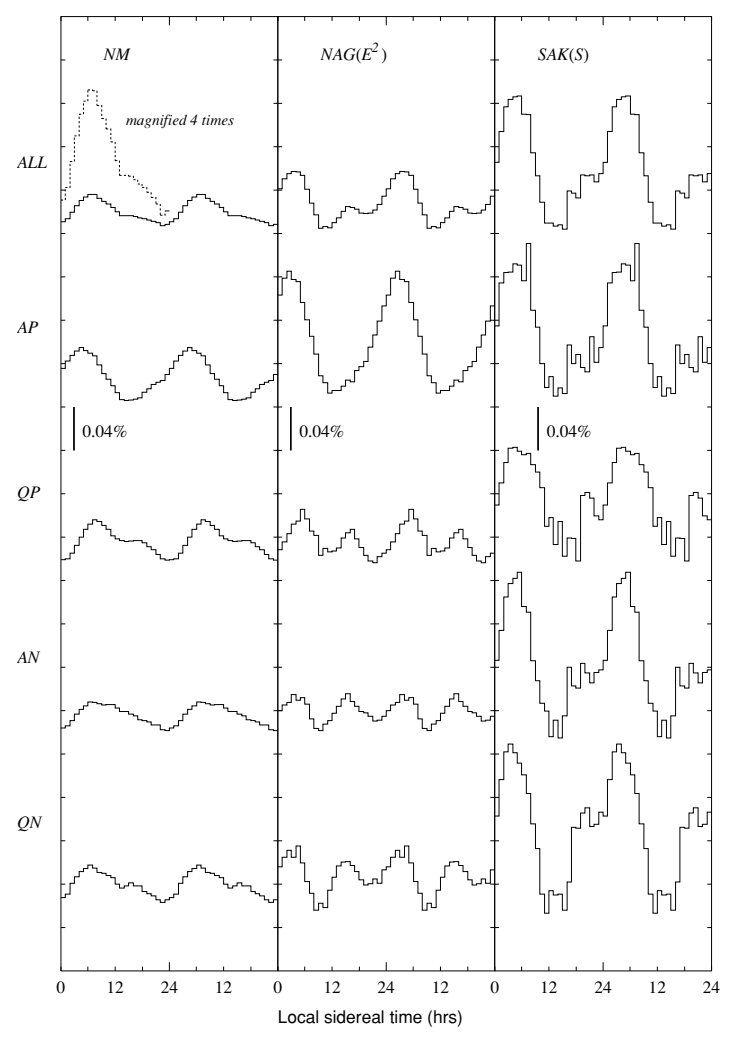

Fig. 14. $I(X, Y) \mathrm{s}$ in $Y=A L L, A P, Q P, A N$ and $Q N$ for $X=N M$ (neutron monitor, $E_{m} \sim 20 \mathrm{GeV}$, cf. Ref. 3), NAG(Nagoya $E^{2}$-telescope, $E_{m} \sim$ $192 \mathrm{GeV}$ ), and $S A K$ (Sakashita $S$-telescope, $E_{m} \sim 387 \mathrm{GeV}$, cf. Ref. 2). The dotted curve at the upper left-hand corner is the magnified $I(N M$, $A L L)$ by 4 times in order to emphasize the hump on the right-hand side of the peak at $\sim 6 \mathrm{hr}$.

$Y)$ s and $I(N M, Y \neq A P)$ s satisfy the criterion, showing respectively the existence and nonexistence of $G(t)$. On the other hand, as $I(N A G, Y \neq A P)$ s have their minimum at $\sim 0$ $\mathrm{hr}$ in the $Q P$ period and $\sim 12 \mathrm{hr}$ in the $A N$ and $Q N$ periods, the criterion indicates the nonexistence of $G(N A G, Q P)$ and the existence of $G(N A G, A N)$ and $G(N A G, Q N)$, in good agreement with the simulation of the polarity dependence of $G$-anisotropy (cf. Refs. 2 and 3). In the above exceptional cases of $I(N M, A P)$ and $I(N A G, A P)$, the criterion does not apply, since they do not contain any remarkable hump.

As above, the hump of $I(N M, A L L)$ in Fig. 14 contains $H(t)$ but does not contain $G(t)$, on the other hand, that of $I(N A G, A L L)$ in $M I$ region contains not only $H(t)$ but also $G(t)$. In $H I$-energy region, however, it is not certain whether the hump is due to only $G(t)$ or the composite of $G(t)$ and $H(t)$ for the following reason. Although $H(t)$ is not easily recognizable in the present form of the hump, in the previous analysis when $I(S A K, A L L)$ was separated into $G(t)$ and $T(t)$ by a somewhat artificial method with the aid of $G(t)$ observed with the air showers $\left(E_{m} \simeq 2 \cdot 10^{4}\right.$ $\mathrm{GeV})$ at Mt. Norikura, $H(t)$ as the by-product of the analysis happened to be narrowly silhouetted against the background noise of $T(t)$, (cf. Ref. 1). Unfortunately, however, $H(t)$ was unknown until its discovery by the neutron monitors in Ref. 3. For confirmation of its existence in the $H I$ region, further observation is necessary as the silhouetted 


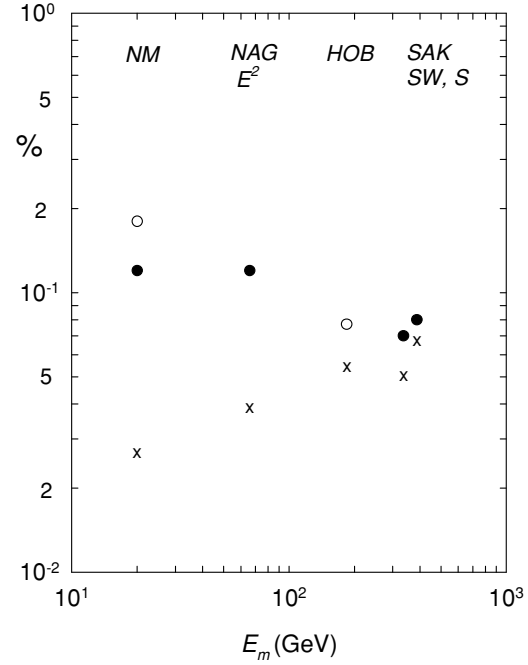

Fig. 15. Energy dependence of $T(X, A P)$ and $T(X, A L L)$ for $X=N M$ (neutron monitor), $N A G$ (Nagoya $E^{2}$-telescope), $S A K$ (Sakashita $S$ - and $S W$-telescopes) and $H O B$ (Hobart, cf. Jacklyn, 1986); • and o: $T(X, A P)$ observed and expected, $\times: T(X, A L L)$ observed.

$H(t)$ is not statistically very significant in this energy region. Similarly, the existence of $H(t)$ in the $M I$-region was also unknown at that time. In the present analysis, the relative contribution of $H(t)$ and $G(t)$ to the hump in the $M I$ region can be estimated as follows. The magnitude of $G(t)$ averaged for 6 years in Fig. 10(b) is about $0.06 \%$ in the equatorial region. If $G(t)$ would have appeared in those 6 years only, its contribution to the hump in the $A L L$ period is $0.012 \%(=0.06 \times 6$ years $/ 29$ years $)$. This value would give the lowest limit of $G(t, A L L)$ as $G(t)$ would be included in other yearly variations in the negative polarity period and also might be submerged even in the positive period. As the averaged magnitude of the humps for $X=S^{3}, S^{2}, S, E^{3}$, $E^{2}, E, S W, S E$ and $V$ measured from the bottom to the top is $\sim 0.023 \%$, the highest limit of $H(t)$ in the $A L L$ period is given by $\sim 0.011 \%$ in the $M I$-region.

The energy spectrum of $T(t)$ has been determined by using the data of the muon telescopes at Nagoya in the previous section. The spectrum can be obtained also by using $T(X, A P)$ s for $X=N M, N A G$ and $S A K$ in wide energy regions. Figure 15 shows the energy dependence of their magnitudes. Two of them, shown by black points, are quoted from Ref. 2 for $X=S A K$ and from Ref. 3 for $\mathrm{X}=N M$, while $T(N A G, A P)$ is substituted by $I(N A G$, $A P)$ in Fig. 14, which does not contain any remarkable $H(t)$ as also seen in $i(S, A P, Z)$ s of Fig. 3. The energydependence of the magnitudes at Nagoya and Sakashita can be explained by $T$-anisotropy just outside the GMS with the energy spectrum in Eq. (1) having $\eta_{0} \simeq 0.43 \%$ and $\gamma_{0} \simeq$ -0.45 , which almost coincides with that $\left(\eta_{0} \simeq 0.36 \%, \gamma \simeq\right.$ $-0.5)$ derived from the first harmonic vectors of $I(X, A P) \mathrm{s}$ in Fig. 8 (cf. Ref. 4). This spectrum produces the expected magnitudes of $T(t)$ s of the neutron monitor and the muon telescope $\left(E_{m}=184 \mathrm{GeV}\right)$ at Hobart $(H O B)$ shown by open circles on the graph (cf. Yasue et al., 1982 and Ref. 4). The value at Hobart is presented for reference, though the corresponding observed value in the $A P$ period is not reported at present. The value for the neutron component is considerably larger than the observation. This difference could be resolved if the spectrum has a low cut-off energy $\left(E_{T C}\right)$ at $\sim 9 \mathrm{GeV}$ below which the $T$-flux becomes zero. Such an energy spectrum with $E_{T C}$ would be produced by solar modulation of the original spectrum with $\gamma=-1.0$ that is supposed to be caused by cosmic-ray acceleration on the boundary of HMS. In the $A L L$-period, on the other hand, the magnitudes of $T(X, A L L)$ s in Fig. 14 are plotted by the cross marks in Fig. 15. The magnitude of $T(H O B$, $A L L$ ) observed at Hobart (Jacklyn, 1986; Ref. 1) is also shown. These data show the rising spectrum of $T(X, A L L)$ with energy in accordance with that obtained in Ref. 1 . The spectrum change from the $A P$ to $A L L$ period is due to the increase of the contamination of $H(X, Y) \mathrm{s}$ in the $Y \neq A P$ period with a decrease of energy.

The energy spectrum of $H$-anisotropy is not certain owing to the domination of $T(t)$ over $H(t)$. As can be seen in $I(X, Y \neq A P) \mathrm{s}, H(t)$ is so small as to be undistinguishable in $I(N M, Y \neq A P)$ s and also almost negligible in $I(S A K, Y$ $\neq A P)$ s even if it were to exist. On the contrary, $H(t)$ in $I(N A G, Y \neq A P)$ is approximately comparable with $T(t)$. These facts indicate that the energy spectrum is not of a simple power type, but must have a low cut-off energy $E_{H C}$ greater than $E_{T C}$ or a maximum in the energy region around $\sim 60 \mathrm{GeV}$. The cause of such a spectral difference between $H$ - and $T$-anisotropies is not certain but would be expected to be due to the difference of the acceleration mechanisms of the cosmic rays on the helionose and heliotail boundaries (cf. Ref. 3).

The right ascensions of the directions of $H$ - and $T$ anisotropies are $\sim 18$ and $\sim 6 \mathrm{hr}$, respectively, on the average but they are variable in time as can be seen in Figs. 3, 4 and 8. The declinations of their directions also would be variable, but their averaged $\delta_{H}$ and $\delta_{T}$ are estimated to be positive and negative respectively on the basis of the following facts as previously pointed out.

1) In Southern high latitudes, the ion chamber at Christchurch $\left(\lambda=44^{\circ} \mathrm{S}\right)$ and the underground muon telescope at Hobart $\left(\lambda=43^{\circ} \mathrm{S}\right)$ cannot observe $H(t)$ but $T(t)$, (cf. Fig. 1 and Jacklyn, 1986).

2) $\Delta I_{\max }(X, Y) \mathrm{s}$ in $Y=Q P$ and $N$ period in Fig. 6 indicate that the latitude distributions of $H(t)$ and $T(t)$ have their maxima in the Northern and Southern Hemispheres, respectively, and the declination $\left(\delta_{H}\right)$ of $H$-anisotropy is estimated at $14^{\circ} \sim 17^{\circ}$.

3) The latitude distribution of $H(t)$ in $i(X, A P, 1991) \mathrm{s}$ in Fig. 7 shows that $\delta_{H}$ of $H$-anisotropy is $24^{\circ} \sim 41^{\circ}$.

4) In the positive $(P)$ period, $I(G O D, P)$ and $I(Y A K$, $P)$ in and close to the Arctic Circle show the maximum phase $(\sim 18 \mathrm{hr})$ of $H(t)$ contrary to that $(\sim 6 \mathrm{hr})$ of $T(t)$ dominating in the Southern hemisphere (cf. Figs. 12 and 13).

These facts support the previous conclusion in Ref. 1 that the major axis $\left(\alpha_{M}=18 \mathrm{hr}, \delta_{M}=24^{\circ}\right)$ of HMS in the noseward direction expected from $T$-anisotropy approximately coincides with that inferred from the direction $\left(\alpha_{P}=18\right.$ hr, $\left.\delta_{P}=29.2^{\circ}\right)$ of the proper motion of the solar system (Campbel and Moore, 1928), but does not coincide with that $\left(\alpha_{N}=16.8 \mathrm{hr}, \delta_{N}=-15^{\circ} \sim-17^{\circ}\right)$ inferred from 


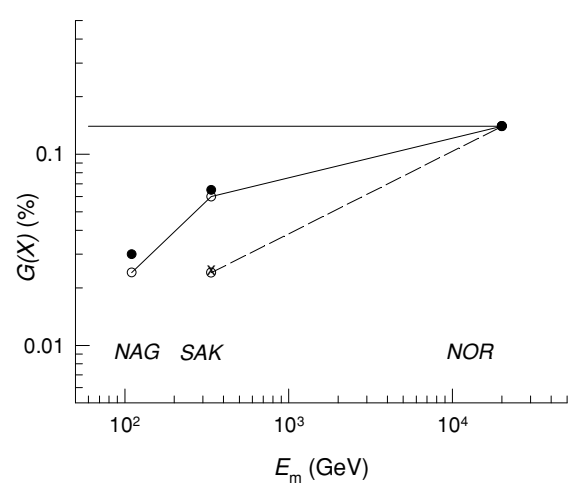

Fig. 16. Solar modulation of the galactic anisotropy, $G(N A G, N)$ at Nagoya in $\mathrm{N}$ period observed (•) and simulated (०). $G(S A K, N)$ at Sakashita $(S W$-telescope) in the $N$ period observed $(\times)$ and simulated (०). $G(N O R, A L L)$ observed with air showers at Mt. Norikura $(\bullet)$ in $A L L$ period. $G(S A K, P)$ at Sakashita ( $S W$-telescope) in the $P$ period observed $(\times)$ and simulated (०). Horizontal line through $G(N O R, A L L)$ expresses the constant energy spectrum of the anisotropy.

the relative motion of the solar system to the neutral gas (McClintock et al., 1978; Ajello, 1978).

The galactic anisotropy is obtained by the comparison of $G(t)$ s with those derived from the simulation of its solar modulation in HMS. The simulation depends on the following factors; the energy spectrum of the anisotropy, the cosmic-ray deflection in the ordered heliomagnetic field (cf. Nagashima et al., 1981b, 1982, 1983), the cosmic-ray scattering mean free path in the irregular field superposed on the ordered magnetic field (cf. Yasue et al., 1985), and the cosmic-ray deflection in GMS and attenuation in the atmosphere (cf. Ref. 4). The black circles in Fig. 16 shows observed $G(t)$ s as a function of the median energy. The one $(\sim 0.03 \%)$ on the left side is the average $(\sim 0.06 \% \times 6$ years/12 years) at Nagoya $\left(E_{m} \simeq 110 \mathrm{GeV}\right)$ in the $N$ period only, during which $G(t)$ is considered to be observed at the station, and the other $(\sim 0.065 \%)$ at the center is the average observed with $S W$-telescope $\left(E_{m} \simeq 336 \mathrm{GeV}\right)$ at Sakashita in the $N$ period (cf. Ref. 2). The black circle on the right side is that $(0.14 \%)$ observed with air showers $\left(E_{m} \simeq 2 \cdot 10^{4} \mathrm{GeV}\right)$ at Mt. Norikura $\left(\lambda=36^{\circ} \mathrm{N}\right.$, $\left.\phi=138^{\circ} \mathrm{E}\right)$ in $A L L$ period, which is almost independent of the solar modulation and is statistically well founded (cf. Nagashima et al., 1989). Two open circles connected with a solid line in Fig. 16 express the simulated $G(t)$ s at Nagoya and Sakashita in $N$ period normalized to $G(N O R, A L L)$, which are obtained on the assumption that the anisotropy has a constant energy spectrum, as shown by the horizontal line through the point $(0.14 \%)$, (cf. Ref. 3 ). There are good coincidences between the observed and expected variations at these two stations in the $N$ period. In addition to this, $G(S A K, P)$ observed with the $S W$-telescope at Sakashita in the $P$ period and shown by the cross mark in Fig. 16 almost coincides with the simulation shown by open circle (cf. Refs. 2 and 3). Although the observations and the simulations contain, respectively, some uncertainties especially in the estimate of the cosmic-ray scattering mean free path (cf. Yasue et al., 1985), their coincidences indicate that the anisotropy would be almost constant in the energy region of $10^{2} \sim 10^{4} \mathrm{GeV}$.

\section{Conclusion}

The three kinds of cosmic-ray sidereal anisotropies called the galactic, helionose-in and heliotail-in anisotropies have been found by the analysis of cosmic-ray sidereal daily variations derived from the long-term observations with the muon telescopes at Nagoya and with the ion chambers at Godhavn, Yakutsk, Cheltenham, Huancayo and Christchurch in the energy regions $(60 \sim 100 \mathrm{GeV})$. The characteristics of the anisotropies have been determined in cooperation with the results observed with the neutron monitors in the low energies $(\sim 20 \mathrm{GeV})$ and with the underground muon telescopes in the high energy regions ( $\sim 200 \sim 700 \mathrm{GeV}$ ). The heliotail-in anisotropy is observable in all the energy regions $(20 \sim 700 \mathrm{GeV})$, the helionose-in anisotropy in the low and middle energy regions $(20 \sim 100$ $\mathrm{GeV}$ ), and the galactic anisotropy is observable in the middle and high energy regions (60 700 GeV).

The galactic anisotropy is subjected to solar modulation and produces a sidereal daily variation larger in the negative-polarity state than in the positive state at Nagoya and Sakashita, showing substantial agreement with the simulation. On the basis of the simulation, the sidereal daily variations observed at Nagoya, Sakashita and Mt. Norikura indicate that the galactic anisotropy has a constant magnitude of $\sim 0.14 \%$ in the energy regions from $\sim 60$ to $\sim 10^{4}$ $\mathrm{GeV}$.

The anisotropies of helioboundary origin observed just outside GMS are variable in their magnitude, direction and energy spectrum, suggesting that the cosmic-ray acceleration is considered to change its activity and its location on the helioboundary surface from time to time, and the accelerated fluxes are subject to variable solar modulation depending on the solar activity and the polarity state of the solar magnetic field. The average right ascension of the helionose-in and heliotail-in anisotropies are $\sim 18$ and $\sim 6$ hr respectively. The average declinations cannot be definitely specified but are positive for the nose-in anisotropy and negative for the tail-in anisotropy. This is in favor of the previous conclusion that the major axis $\left(\alpha_{M}=18 \mathrm{hr}\right.$, $\delta_{M}=24^{\circ}$ ) of HMS in the noseward direction expected from the heliotail-in anisotropy does not coincide with that $\left(\alpha_{N}=16.8 \mathrm{hr}, \delta_{N}=-15^{\circ} \sim-17^{\circ}\right)$ inferred from the relative motion of solar system to the neutral gas but approximately coincides with that $\left(\alpha_{P}=18 \mathrm{hr}, \delta_{P}=29.2^{\circ}\right)$ inferred from the proper motion of solar system. The energy spectrum of the average heliotail-in anisotropy is of the power-exponent type with $\gamma_{0} \simeq-0.45 \sim-0.5$ and with the low cut-off energy $\left(E_{T C}\right)$ at $\sim 9 \mathrm{GeV}$, whereas that of the helionose-in anisotropy is also of power-exponent type with negative $\gamma$ but with the low cut-off energy $E_{H C}$ greater than $E_{T C}$ or has a peak in the energy region of $\sim 60 \mathrm{GeV}$.

Finally, solar modulation of the cosmic-ray sidereal daily variation of helioboundary origin would provide important information as to the cosmic-ray acceleration mechanism and the propagation of the accelerated fluxes in HMS. One of the most important findings is the predominance of the heliotail-in anisotropy in the $A P$ period (Active period of solar cycle in Positive polarity state), which suggests that the cosmic rays are most actively accelerated on the polar region $\left(|\lambda|>>0^{\circ}\right)$ of the cylindrical surface of the helio- 
tail boundary, especially in the active period, and that they arrive at the Earth only in the positive polarity state.

Acknowledgments. The authors express their sincere appreciation to the late Dr. S. E. Forbush of the Carnegie Inst. of Washington and also to the late Professor Emeritus Yu. G. Shafer and Dr. and Mrs. G. B. Shafer of the Yu. G. Shafer Institute of Cosmophys. Research and Aeronomy, Yakutsk, who were responsible for the publication of the long-term ion-chamber data, used here. The authors express their deepest gratitude to the late Professor Emeritus Y. Sekido, the founder of Cosmic-Ray Research Laboratory, Nagoya University, who was responsible for setting up the Nagoya and Sakashita muon telescopes and also to the late Professor Emeritus H. Ueno who was responsible for subsequent unique length of continuous output from the telescope systems in cooperation with one (Z.F.) of the present authors. The authors thank M. Orito and T. Yamada and Mrs. H. Satake for the maintenance of the Nagoya muon telescope and for processing the muon data over a long period. The authors are grateful to Dr. R. M. Jacklyn, Antarctic Division, Department of Science and Technology, Australia, Professor Emeritus I. Kondo, Institute for Cosmic Ray Research, University of Tokyo, and the members of Cosmic-Ray Section, Solar-Terrestrial Environment Laboratory, Nagoya University for their kind discussions.

\section{References}

Ajello, J. M., An interpretation of Mariner 10 helium (584 $\AA$ ) and hydrogen $(1216 \AA)$ interplanetary emission observations. Astrophys. J. 222, 1068 1078, 1978.

Beach, L. and S. E. Forbush, Cosmic-ray Results, Publ. 175, Vol. XXI, Carnegie Inst. of Washington, Washington, D.C., 1961.

Campbel, W. W., and J. H. Moore, Publ. Lick Obs., 16, 38, 1928.

Fujii, Z., S. Sakakibara, K. Fujimoto, and H. Ueno, Multi-directional Cosmic-ray Intensities, Nagoya, Report of Cosmic-Ray Research Laboratory, No. 15, Cosmic-Ray Research Laboratory, Nagoya Univ., Nagoya, Japan, 1990.

Fujii, Z., S. Sakakibara, K. Fujimoto, H. Ueno, M. Orito, and T. Yamada, Multi-directional cosmic-ray intensities, Nagoya, Report of Cosmic-Ray Research Section, Nos. 17, 18, Cosmic-Ray Research Section, SolarTerrestrial Environment Laboratory, Nagoya Univ., Nagoya, Japan, 1993, 1996.

Fujii, Z., S. Sakakibara, K. Fujimoto, M. Orito, and T. Yamada, Multidirectional cosmic-ray intensities, Nagoya, Report of Cosmic-Ray Research Section, No. 19, Cosmic-Ray Research Section, Solar-Terrestrial Environment Laboratory, Nagoya Univ., Nagoya, Japan, 2000

Fujimoto, K., A. Inoue, K. Murakami, and K. Nagashima, Coupling coefficients of cosmic-ray daily variations for muon telescopes, Report of Cosmic-Ray Research Laboratory, No. 9, Cosmic-Ray Research Laboratory, Nagoya Univ., Nagoya, Japan, 1984.

Howard, R., Studies of solar magnetic fields I: The average field strength. Solar Phys. 38, 283-299, 1974.

Inoue, A., M. Wada, and K. Kondo, Asymptotic direction in 1975, Cosmic Ray Table, No. 1, WDC-C2 for Cosmic Rays, Institute of Phys. and Chem. Res., Tokyo, 1983.

Jacklyn, R. M., Galactic cosmic ray anisotropies in the energy range $10^{11}$ $10^{14}$ eV., Proc. Astron. Soc. Australia, 6, 425-436, 1986.
Lange, I. and S. E. Forbush, Cosmic-ray results, Publ. 175, Vol. XIV, Carnegie Inst. of Washington, Washington, D.C., 1948.

Lange, I. and S. E. Forbush, Cosmic-ray results, Publ. 175, Vol. XX, Carnegie Inst. of Washington, Washington, D.C., 1957.

McClintock, W., R. Henry, J. L. Linsky, and H. Moos, Ultraviolet observations of cool stars, II. Local interstellar hydrogen and deuterium Lymanalpha. Astrophys. J., 225, 465-481, 1978.

Nagashima, K., Z. Fujii, S. Sakakibara, K. Fujimoto, and H. Ueno, Multidirectional cosmic-ray intensities, Nagoya, Report of Cosmic-Ray Research Laboratory, Nos. 3, 4, 10, 11, Cosmic-Ray Research Laboratory, Nagoya Univ., Nagoya, Japan, 1978 (No. 3), 1981a (No. 4), 1984 (No. 10), 1987 (No. 11)

Nagashima, K. and I. Morishita, Cosmic ray sidereal daily variation of galactic origin observable in heliomagnetosphere, Report of CosmicRay Research Laboratory, Nagoya Univ., Nagoya, Japan, No. 8, 1983.

Nagashima, K., I. Morishita, and S. Yasue, Asymptotic orbit of cosmic rays incident on the Earth from galactic space, Proc. 17th International Cosmic Ray Conference, 4, 189-192, 1981b.

Nagashima, K., I. Morishita, and S. Yasue, Modulation of galactic cosmic ray anisotropy in the heliomagnetosphere: Average sidereal daily variation, Planet. Space Sci., 30, 879-896, 1982.

Nagashima, K., K. Fujimoto, S. Sakakibara, Z. Fujii, H. Ueno, I. Morishita, and K. Murakami, Galactic cosmic-ray anisotropy and its modulation in the heliomagnetosphere, inferred from air shower observation at Mt. Norikura, Nuovo Cimento, 12C, No. 6, 695-749, 1989.

Nagashima, K., K. Fujimoto, and R. M. Jacklyn, Cosmic ray sidereal daily variation showing the coexistence of the galactic and heliomagnetotailin anisotropies, Proc. 24th International Cosmic Ray Conference, 4, 652-655, 1995a.

Nagashima, K., K. Fujimoto, and R. M. Jacklyn, Cosmic ray excess flux from heliomagnetotail, Proc. 24th International Cosmic Ray Conference, 4, 656-659, 1995b.

Nagashima, K., K. Fujimoto, and R. M. Jacklyn, Galactic and heliotail-in anisotropies of cosmic rays as the origin of sidereal daily variation in the energy region $<10^{4} \mathrm{GeV}$, J. Geophys. Res. 103, 17429-17440, 1998.

Nagashima, K., Z. Fujii, and K. Munakata, Solar modulation of galactic and heliotail-in anisotropies of cosmic rays at Sakashita underground station (320 650 GeV), Earth Planets Space, 56, 479-483, 2004.

Nagashima, K., I. Kondo, and Z. Fujii, Sharply concentrated cosmic-ray excess fluxes from heliomagnetoshperic nose and tail boundaries observed with neutron monitors on the ground, Earth Planets Space, 57, 1083-1091, 2005.

Sekido, Y., K. Nagashima, I. Kondo, H. Ueno, K. Fujimoto, and Z. Fujii, Multi-directional cosmic-ray intensities, Nagoya, Report of Cosmic-Ray Research Laboratory, No. 1, Cosmic-Ray Research Laboratory, Nagoya Univ., Nagoya, Japan, 1975.

Shafer, G. B. and Yu. G. Shafer, Precise observation of cosmic rays at Yakutsk, Institute of Cosmophysical Research and Aeronomy, Yakutsk, Science Academy of USSR, 1984.

Yasue, S., S. Mori, S. Sakakibara, and K. Nagashima, Coupling coefficients of cosmic ray daily variations for neutron monitor stations, Report of Cosmic-Ray Research Laboratory, No. 7, Cosmic-Ray Research Laboratory, Nagoya Univ., Nagoya, Japan, 1982.

Yasue, S., I. Morishita, and K. Nagashima, Modulation of galactic cosmic ray anisotropy in heliomagnetosphere: influence of cosmic ray scattering on sidereal daily variation, Planet. Space Sci., 33, 1057-1068, 1985.

K. Nagashima and Z. Fujii (e-mail: fujii@stelab.nagoya-u.ac.jp) 